\title{
Review
}

\section{Monitoring the levels of important nutrients in the food supply}

\author{
B. Neal ${ }^{\# \#}$, G. Sacks ${ }^{2 \#}$, B. Swinburn",3\#, S. Vandevijvere ${ }^{3 \#}$, E. Dunford ${ }^{1 \#}$, W. Snowdon $2,4 \#$, J. Webster ${ }^{1 \#}$, \\ S. Barquera ${ }^{5}$, S. Friel ${ }^{6}$, C. Hawkes ${ }^{7}$, B. Kelly ${ }^{8}$, S. Kumanyika9 ${ }^{9}$, M. L'Abbé ${ }^{10}$, A. Lee ${ }^{11,12}$, T. Lobstein ${ }^{13,14}$, \\ J. Ma ${ }^{15}$, J. Macmullan ${ }^{16}$, S. Mohan ${ }^{17}$, C. Monteiro ${ }^{18}$, M. Rayner ${ }^{19}$, D. Sanders ${ }^{20}$ and C. Walker ${ }^{21}$ \\ for INFORMAS^
}

\footnotetext{
${ }^{1}$ The George Institute for Global Health, University of Sydney, Sydney, New South Wales, Australia; ${ }^{2}$ WHO Collaborating Centre for Obesity Prevention, Deakin University, Melbourne, Victoria, Australia; ${ }^{3}$ School of Population Health, University of Auckland, Auckland, New Zealand; ${ }^{4}$ Pacific Research Centre for the Prevention of Obesity and Non-communicable Diseases (C-POND), Suva, Fiji; ${ }^{5}$ National Institute of Public Health, Mexico City, Mexico; ${ }^{6}$ National Centre for Epidemiology and Public Health, Australian National University, Canberra, Australian Capital Territory, Australia; ${ }^{7}$ World Cancer Research Fund International, London, United Kingdom; ${ }^{8}$ School of Health and Society, University of Wollongong, Wollongong, New South Wales, Australia; ${ }^{9}$ Perelman School of Medicine, University of Pennsylvania, Philadelphia, Pennsylvania, United States; ${ }^{10}$ Department of Nutritional Sciences, University of Toronto, Toronto, Canada; ${ }^{11}$ School of Public Health and Social Work, Queensland University of Technology, Brisbane, Queensland, Australia; ${ }^{12}$ School of Exercise and Nutrition Sciences, Queensland University of Technology, Brisbane, Queensland, Australia; ${ }^{13}$ International Association for the Study of Obesity, London, United Kingdom; ${ }^{14}$ Public Health Advocacy Institute of Western Australia, Curtin University, Perth, Western Australia Australia; ${ }^{15} \mathrm{Chinese}$ Center for Disease Control and Prevention (CCDC), Beijing, China; ${ }^{16}$ Consumers International, London, United Kingdom; ${ }^{17}$ Public Health Foundation of India, New Delhi, India; ${ }^{18}$ School of Public Health, University of Sao Paulo, Sao Paulo, Brazil; ${ }^{19}$ British Heart Foundation Health Promotion Research Group, University of Oxford, Oxford, United Kingdom; ${ }^{20}$ School of Public Health, University of the Western Cape, Cape Town, South Africa; ${ }^{21}$ Global Alliance for Improved Nutrition (GAIN), Geneva, Switzerland
}

\begin{abstract}
Summary
A food supply that delivers energy-dense products with high levels of salt, saturated fats and trans fats, in large portion sizes, is a major cause of non-communicable diseases (NCDs). The highly processed foods produced by large food corporations are primary drivers of increases in consumption of these adverse nutrients. The objective of this paper is to present an approach to monitoring food composition that can both document the extent of the problem and underpin novel actions to address it. The monitoring approach seeks to systematically collect information on high-level contextual factors influencing food composition and assess the energy density, salt, saturated fat, trans fats and portion sizes of highly processed foods for sale in retail outlets (with a focus on supermarkets and quick-service restaurants). Regular surveys of food composition are proposed across geographies and over time using a pragmatic, standardized methodology. Surveys have already been undertaken in several high- and middle-income countries, and the trends have been valuable in informing policy approaches. The purpose of collecting data is not to exhaustively document the composition of all foods in the food supply in each country, but rather to provide information to support governments, industry and communities to develop and enact strategies to curb food-related NCDs.
\end{abstract}

Keywords: Food composition, INFORMAS, monitoring, processed food.

obesity reviews (2013) 14 (Suppl. 1), 49-58

Address for correspondence: B Neal, The George Institute for Global Health, The University of Sydney, PO Box M201, Missenden Rd, NSW 2050, Australia.

E-mail: bneal@georgeinstitute.org.au

\footnotetext{
"Members of the writing group for this manuscript are listed in order of their contribution to the writing of the manuscript. IINFORMAS is the International Network for Food and Obesity/non-communicable diseases Research, Monitoring and Action Support. All authors who are not members of the writing group are listed in alphabetical order, and contributed to discussion of the key concepts and issues raised in this manuscript as part of the first formal meeting of INFORMAS from 19 to 23 November 2012.
} 


\section{Background}

A nation's food supply is typically comprised of a wide range of different types of foods produced by a diverse group of providers spanning small holders, agribusiness, national and international food manufacturers, and retailers. Although there is still much variation between countries, the process of globalization is driving rapid change in the nature of the world's food supply, particularly in those regions currently undergoing rapid economic development and social transition (1-4). The result is a progressive shift away from the consumption of fresh foods prepared at home towards a diet based upon consumer packaged goods and foods eaten out of the home (5-8). Accompanying this transition, and in substantive part driven by the population-wide change in average diet, has been an explosion of non-communicable diseases (NCDs) (9).

A primary vector of this epidemic is the large food corporation and the highly processed foods that it provides (10). There are now few countries unaffected by the many unhealthy commodities purveyed by these corporations, and while the markets for highly processed foods in developed countries are mostly saturated, the food industry projects substantial growth in both market size and profitability for many low- and middle-income countries (10). Further, large increases in obesity, dyslipidaemia, high blood pressure, diabetes and the risks of premature death from vascular disease and cancer will be a direct consequence of expanded sales of highly processed foods $(10,11)$.

While there is a very clear understanding that the products delivered by these corporations are seriously detrimental to health (12), there has been little action to curb the harms caused. In part, this is because there is an absence of data to adequately define the extent of the problem and the actions that might be taken to ameliorate it. Until recently, most efforts to track the nature of the food supply have been focused upon understanding micronutrient deficiencies and under-nutrition with the primary objective being to ensure adequate production and distribution of commodities such as rice, beans, wheat and meat. Currently, however, the primary issue is one of excess consumption of foods high in salt, saturated fat, trans fat and energy that are causing a different burden of diet-related ill health (9).

In 2004, the World Health Organization's Global Strategy on Diet, Physical Activity and Health recommended that the food industry limit the levels of saturated fatty acids, trans fatty acids, free sugars and salt in their existing processed food products (13). As a direct consequence, a number of governments and large corporations have acknowledged the role they can play and announced strategies to improve the food supply. Some large corporations have reported improvements in their product range $(14,15)$, although most have made either no commitment or are failing to take actions promised (10). Likewise, there are few governments that have taken substantive action, and governments are largely relying upon voluntary commitments and self-regulation by the industry.

The International Network for Food and Obesity/noncommunicable diseases Research, Monitoring and Action Support (INFORMAS) is a global network of publicinterest organizations and researchers that aims to monitor, benchmark and support public and private sector actions to create healthy food environments and reduce obesity, NCDs and their related inequalities (16). This paper introduces the food composition module of INFORMAS, which seeks to answer the research question, 'What is the composition of foods and non-alcoholic beverages (henceforth, just "foods")?". The objective of this paper is to define approaches to the collection of systematic and directly comparable information on food composition, and show how this can be linked in with other food environment monitoring activities and translated for use in informing policies and action.

\section{Overall approach to monitoring food composition}

There are a range of information sources, that have been designed for a variety of different purposes, that already provide data about the composition of foods (17). For example, household expenditure surveys and national nutrition surveys (18) which use food composition data have made it possible for governments and other interest groups to track various aspects of the nutritional adequacy of a population's diet. Few of these existing sources of information on food composition, however, provide the type of information that can be used to properly characterize and monitor the actions of the large corporations that are now transforming cultural dietary norms and changing the tastes of whole populations (19).

The first component of the proposed monitoring approach is a contextual analysis that determines the status of existing policies and actions influencing food composition, and assesses how the work proposed in this module can best complement current knowledge and activities. This will include a review of government, industry, nongovernment organization and consumer actions to improve the nutrient profile of foods in the food supply, as well as the data used to inform the development and evaluation of those initiatives.

Assuming that, after the initial contextual analysis, the collation of additional information about food composition is considered worthwhile then a phased approach is proposed, with the extent of implementation dependent upon the resources available. The work plan proposed here builds upon a related existing initiative being carried out by an established network active in this field, the Food 
Monitoring Group (20-22). That collaboration currently involves some 30 countries, has a number of established protocols and tools, has defined processes for data management and already holds substantive data for a number of countries (23-25). Much of the development work done for the Food Monitoring Group can be applied directly to the objectives of this INFORMAS food composition module.

Once the broad context is understood, the second component involves making a high-level estimate of the proportion of the food supply that consumers in the country derive from different sources - principally home production, retail outlets (such as supermarkets, corner or convenience stores, vending machines, fresh produce markets), workplaces, schools and restaurant outlets (including quick-service restaurants, fast-food outlets and any other outlet selling ready-to-eat snacks and meals). The data to make this estimate will likely be derived from household budget surveys, population dietary surveys and industry reports. Where possible, it will be valuable to have information about the relative contribution of small-to-medium enterprises versus large national and transnational corporations when quantifying the food derived from retail and restaurant outlets. It is recognized that there may be significant variation in the composition of the food supply between settings within a country and that data may, for example, need to be considered separately for urban, rural and slum areas. The goal of this second component is not to exhaustively define the composition of the food supply, but rather to obtain the insight required to appropriately direct subsequent data collection efforts and inform policy responses.

The third component of the proposed monitoring approach is large-scale data collection and analysis. The focus of this component will be guided by the findings of the first two components, with prioritization given to the collection of those data that will most likely support change. For many countries, where there are limited data to define the nature of the foods provided by large corporations, this will likely involve surveys of the numbers and types of foods available, and the collection of nutrient composition data reported on food labels or in other documentation. These data can be used: to identify those areas of the food supply most urgently requiring action; for benchmarking and target setting; as well as to hold individual corporations directly to account for the composition of their products. If possible, metrics that describe the sales volume of each product can be collected, although these data may be challenging to procure.

The final component, to be done where resources are available, is to validate the nutrition information collected from food labels and other secondary data sources against the results of direct chemical analyses of a sample of food products.

\section{Specific methods}

The four main components of this module, as outlined above (contextual analysis; evaluation of the main sources of foods consumed by the population; documentation of food types and the nutrient composition of available foods; and validation of the quality of the food composition data by direct testing), make use of qualitative and quantitative research methodologies. The intent is that the same basic methodology is applied in each country and at each time point such that robust comparisons can be made between data collected in different settings and over time.

\section{Contextual analysis of influences on food composition}

A contextual analysis, based upon an examination of existing records and discussions with stakeholders, is the first component of the proposed monitoring approach. The research can be commenced by reviewing government, industry and other data sources to document the key statutory, commercial and cultural factors influencing food composition in the country. The regulatory analysis can largely be achieved using Internet-based searches of online databases and websites. In many countries, this will uncover regulations related to the declaration of the nutrient content of foods, and, in some countries, there will be records of voluntary industry commitments and ongoing actions by consumer and other non-governmental organizations. Where additional resources are available, these initial searches can be supplemented with a stakeholder analysis done by interviewing a purposive sample of individuals with roles in the food supply. This should include representatives from government departments (such as health, trade, commerce and agriculture), the food industry (production, processing and retail) and civil society (consumer organizations and advocacy groups). A thematic analysis of the data collected from the stakeholders should provide the required insight into the main factors influencing food composition, as well as helping to identify the best opportunities for improving the composition of the food supply (26).

\section{Identifying the main sources from which consumers obtain food}

It is important to understand the main food sources for the population in order to establish the need for additional data collection. For example, if the great majority of the diet were found to derive from foods grown at home, the collection of data about processed or restaurant foods may contribute little to understanding the current context (although it may provide important baseline data as the population goes through nutrition transition). Likewise, if a significant proportion of the diet is from restaurant foods, 
it will be important to understand whether the main providers are large-chain restaurants or small owner-operator type outlets. Data derived from existing household budget surveys, population dietary surveys and industry reports are likely to provide the information required for this component, and the information can be sourced through standard Internet searches or direct communication with relevant experts. It will be important to consider whether there is significant variation in the sources of foods between different geographies and societal groups when making the national estimates. If possible, broad quantitative estimates of the numbers of different types of outlets in the country and the numbers of outlets operated by major national or international chains should be recorded.

\section{Documenting the numbers, types and composition of foods}

With appropriate insight into the sources via which consumers acquire foods, and the potential for new data on food composition to influence the healthiness of the foods available, it will be possible to define a data collection strategy tailored to the country's needs. In most countries, this is likely to focus upon attaining a better description of processed and restaurant foods. A number of countries have adopted a common approach to the collection of such data based upon systematic surveys of the products available in a diverse range of food outlets using an existing standardized methodology (20). Detailed documentation of the contribution that processed and restaurant foods make to the food supply has been instrumental in defining the nature of food supply issues, enabling benchmarking and target-setting, and providing for objective tracking of the effectiveness of food supply interventions over time $(27,28)$. Outputs from the data have also proved an effective means of highlighting food supply issues to a diverse range of stakeholders through reports made in the specialist and general media.

\section{Food outlets evaluated}

Broadly, there are two means by which the community typically accesses foods that are not produced at home: retail outlets (such as supermarkets, corner or convenience stores, vending machines, fresh produce markets) and restaurant outlets (including quick-service restaurants, fastfood outlets and any other outlet selling ready-to-eat snacks and meals). In most countries, there is a diverse range of both retail and restaurant outlets, and this should be defined within the second component of the proposed approach (described above). Based upon this knowledge, a sampling strategy can be developed to ensure that samples of retail and/or restaurant outlets are identified for surveying. In practice, this is likely to mean the inclusion of a range of stores extending from large chains to sole traders with the goal being to include at least one outlet for all major national and international corporations as well as each of the different types of local stores operating in the country. The intent of the survey is not to obtain a weighted sample of all outlets but rather to sample outlets in such a way that the breadth of available products is captured. This will mean selecting single outlets of each type/brand until the majority of the providers have been covered. Where there is significant geographic disparity in the types of outlets, it may be necessary to survey at multiple sites. However, for many countries, surveys done within a single region are likely to capture much of the diversity.

\section{Foods included}

The foods included in the survey will be every product available for sale in the food outlets selected for evaluation. It is expected that products will mostly be identified from surveys done in-store at retail and restaurant outlets. For large-chain restaurants, it is possible that data will be more easily collected from Internet searches of company websites. Likewise, some countries may be able to use social media and 'crowd-sourcing' techniques to support the collection of data for processed food products (see Box 1). The goal will be to collect data using an easily repeatable methodology with experts within the countries determining the most feasible way to collect data based on available resources and local circumstances. A primary consideration will be the future repetition of the methods such that temporal changes can be reliably determined. While the recommendation is to list and collect data on every food item for sale in selected retail outlets, it may be that groups conducting monitoring in some countries elect to undertake a sampling approach.

\section{Information to be collected about food composition}

The primary ('minimal') and secondary ('expanded') information that will be sought for each food product is indicated (Table 1). Missing data will be recorded as such, and products for which only company name and product name are available with no nutrition data will be noted in order to highlight the absence of data. Documenting the absence of food composition data will be an important objective and will be done in an effort to drive policy changes towards enforced standardized nutrient labelling of foods. It is anticipated that there will be five main sources of information:

- Data obtained from the 'nutrient declarations' on product labels

- Data obtained from Internet searches of corporate websites

- Existing food composition databases 


\section{Box 1 'Crowd-sourcing' using the FoodSwitch smartphone application}

The 'FoodSwitch' smartphone application was launched by the George Institute for Global Health in Australia in collaboration with the health insurer, Bupa. The application allows consumers to use the phone camera to scan the barcodes of processed food products and receive on-screen a multiple traffic-light label showing the levels of fat, saturated fat, sugar, salt and energy in the product. In addition, the application scans the database for similar foods, and, based on a nutrient profiling score, makes recommendations about healthier alternatives that the user might want to purchase instead.

FoodSwitch was launched with a database of about 12,000 products behind it although it was known that there were more than 50,000 processed food products for sale in Australia. Included in the application was the opportunity for users to send in data by taking photos of the item if the scanned barcode did not map to a product in the database. These products would then be added to the database as an update.

On the day of release in February 2012, FoodSwitch was the most popular free iPhone application in Australia. It remained one of the top 10 most popular free health and fitness applications in the iTunes store for most of the year, and has been downloaded by more than 350,000 Australians. Most striking of all has been the willingness of users to send in missing data. Within 2 days of release, the George Institute had received 26,000 photos of missing items, and the Institute continue to receive about 500 hundred photos every day of new items launched into the market.

FoodSwitch started as a tool for providing data to consumers, but has now become a method for data collection. Australia now has a complete and contemporary database thanks to the support received from the application users. It is likely that the same approach could be used in many other countries. Furthermore, with planned upgrades to the supporting software that will enable optical character recognition and automated categorization of foods, the maintenance resources required are likely to decline substantially.

- Data provided directly by manufacturers

- Data determined from direct chemical analysis of products

Where data is obtained from product labels, it will likely be valuable to tie data collection processes in with data collection for other INFORMAS modules, such as the food labelling (29) and food price (30) modules.
Table 1 Proposed measurement indicators

\begin{tabular}{ll}
\hline & Format \\
\hline Primary ('minimal') & \\
Country & \\
Food group & Country where data is collected \\
Food category & As defined \\
Food subcategory & As defined \\
Company name & As defined \\
Product name & As per data source \\
Serving size & As per data source \\
Energy density & Grams or millilitres \\
& Kilojoules or kilocalories/100 grams or \\
Saturated fat & 100 millilitres \\
Trans fat & Grams/100 grams or 100 millilitres \\
Sodium* & Grams/100 grams or 100 millilitres \\
Data source & Milligrams/100 grams or 100 millilitres \\
Date of data collection & NIP, MANUF, WEB, DATAB, Other \\
Secondary ('expanded') & Date (dd/mm/yyyy) \\
Monounsaturated fat & \\
Polyunsaturated fat & Grams/100 grams or 100 millilitres \\
Added sugars & Grams/100 grams or 100 millilitres \\
Total sugars & Grams/100 grams or 100 millilitres \\
Total fat & Grams/100 grams or 100 millilitres \\
Protein & Grams/100 grams or 100 millilitres \\
Carbohydrate $/ 100$ grams or 100 millilitres \\
Dietary fibre \\
Ingredients list
\end{tabular}

*This can also be recorded as salt in grams/100 grams or 100 millilitres.

DATAB, from external branded database; MANUF, direct from manufacturer; NIP, nutrition information panel on product packaging;

WEB, direct from Internet site.

The direct chemical analysis of products is resource intensive, and will be beyond the scope of monitoring efforts in most countries. However, the comparison of data from direct analysis against data obtained from the nutrition panels of food labels will be attempted for a sample of products in some countries with adequate resources to establish the validity of the food label data.

\section{Categorization of foods}

Foods will be classified in an agreed hierarchical structure to food 'groups', 'categories' and 'subcategories'. The overarching goal for the categorization system will be that it is applicable across multiple countries and settings $(25,31)$, and serves the multilevel monitoring purposes of INFORMAS, taking into account monitoring frameworks for other modules, such as the food labelling (29) and food prices modules (30). It is anticipated that the categorization will be based on systems established for comparable food composition databases $(17,32-35)$ while also reflecting industry practices and consumer purchasing patterns. This 
will be important for enabling reporting that is easily interpretable by industry, government and other stakeholders. It is recognized that some food types will be specific to particular countries or regions, and there will be flexibility within the categorization system to accommodate this. Efforts will be made to have the same structure to the categorization at a high level (e.g. 'breads') but to allow for variation in the subcategorization (e.g. the number of bread subcategories may differ from one setting to another). Existing systems, primarily Codex (36), will be the reference for the categorization system.

\section{Practicalities and required resources}

The goal will be to have an annual cycle of data collection, although less frequent intervals will be acceptable if resources are constrained. Data collected every few years will be adequate to track trends in the composition of the food supply, although may be less effective in terms of driving change.

The collation of the database comprises three main steps. First, the collection of information; second, the entry of that information into a database; and third, the cleaning and analysis of the information. There are a number of tried and tested tools available to facilitate this process including smartphone applications for data collection, and software tools for data processing (20-22,37-40). By way of example, the annual collation of the approximate 7,000 food items initially included in the Australian database required about $400 \mathrm{~h}$ of data collector time, $500 \mathrm{~h}$ of data entry time and $500 \mathrm{~h}$ for categorization and checking of the data. The process followed was one in which data entry staff visited stores and systematically photographed every food item using a smartphone application. The smartphone automatically uploaded the photographs to a software system which allowed data entry staff to transcribe the information on the photographs into a database. The entered data were then checked, and each item assigned to one of about 600 food subcategories in the final database. Considerable streamlining of this approach and significant reductions in the resourcing required are anticipated with the introduction of optical character recognition and automated food categorization methods currently under development.

For those with the resources required, the tools and methods utilized are available on the Food Monitoring Group website (http://www.georgeinstitute.org/projects/ global-food-monitoring-group). It is of note that the initial collection of data in the first year is the most time-consuming piece of work with subsequent years requiring less resources since a substantial number of products carry over from one year to the next. If data can be downloaded in a useable electronic format from other sources then the process might be substantially abbreviated. Likewise, the process may be quicker for restaurant foods where data may be accessed from Internet sources in tabular formats that are quicker to add to the database. More recently, the Australian team have used smartphone application-based crowd-sourcing techniques to minimize ongoing data collection costs while expanding the scope of the data collection process (see Box 1).

\section{Analysis and reporting of food composition data}

There will be tabulations that summarize the numbers of products and the completeness of data, overall, for each country, for each grouping of food types, for types of food outlets and for major companies. The nutrient analysis will be for the key measurement indicators (Table 1) with reporting and exploration of the findings done in the same way as for the examination of the completeness of the data. Mean levels (and ranges) for nutrient values will be calculated per $100 \mathrm{~g}$ and per serve with comparisons made using statistical tests appropriate to the distribution of the data. Benchmarks will be identified and proportions of products meeting targets will also be estimated. Food category targets for nutrient levels, energy density and portion size will be developed by exploring the range of values in each food category and identifying levels that have already been achieved by a significant proportion of products in the category. In regard to testing for differences, there will be no formal adjustment strategy used to control for the many possible analyses that will be done. Instead, individual findings will be interpreted in light of the patterns observed across the broader data set and the number of comparisons made.

\section{Measurement indicators and proposed targets}

The proposed measurement indicators have been selected on the basis that they are likely both to be important causes of diet-related ill health (9), and to be reasonably widely reported across a range of food types and geographic settings. It is a reflection of the nature of most nutrition labelling, and the associated Codex recommendations (36), that the proposed indicators are micronutrients, macronutrients and energy density. This does not preclude countries for which other useful forms of data are available from collecting and using them, but rather, the approach proposed here attempts to provide a common base data set upon which comparisons between different parts of the world's supply of foods can be made.

The primary ('minimal') measurement indicators proposed are saturated fat, trans fat, sodium, energy density and serving size. Secondary ('expanded') measurement indicators are total fat, monounsaturated fat, polyunsaturated fat, protein, total sugars, carbohydrate and dietary fibre. It is recognized that there will be some overlap in the health effects captured by these indicators (9) - for 
example, the effects of saturated fat and polyunsaturated fat are, broadly speaking, likely to be opposite in direction but closely correlated, and one might argue that data on just one or the other should be collected. However, the full set has been included because all have a reasonable evidence base behind them (13) and all are likely to be fairly widely reported (36). In addition, some data might be missing in one region but present in another.

A number of countries have set targets for acceptable levels of nutrients and energy in foods (27). The completeness of coverage of the food supply, the groupings of foods for which targets have been set and the methods used to set these targets have varied (41). Most approaches to target setting have included an empirical component to the process, although most appear to have also been subject to qualification through industry participation (41). Targets do, however, appear likely to be an important component of success in driving change in the composition of the food supply $(41,42)$. Accordingly, as part of the overall INFORMAS programme of work associated with this module, it is envisaged that a systematic assessment of prior target-setting exercises will be conducted, and recommended targets for nutrient and energy levels for all specified food categories, based on a primarily empirical process, will be developed and adjusted over time.

\section{Discussion}

To date, there has been little success in efforts to stem the tide of diet-related ill health that is attributable to burgeoning global sales of highly processed foods containing high levels of adverse nutrients (10). Central to the challenge faced by government and public health is the absence of good data by which to properly define, understand or respond to the problem. The global nature of the issue, and the enormous size and reach of the corporations responsible, requires an equally substantive response, and it is with this in mind that this component of the INFORMAS initiative has been formulated. The project is premised upon the observation that while many countries have a high-level insight into the nature of their food supply, few have the detailed information required to understand the contribution made by different sectors. This, in turn, makes it difficult for governments, industry and community groups to develop and implement adequately targeted interventions, and to track their effectiveness once they have been put in place.

Nutritional transition towards diets based upon highly processed food products is widely understood to be a cause of NCDs in many countries. However, in itself, this knowledge has proved universally inadequate to drive a reduction in the ill health caused by these types of commodities. Indeed, in many countries that have identified the consumption of highly processed foods as a health problem, the attributable disease burden continues to grow (9). In the few countries where there is evidence of success, a key component has been a detailed plan that defines the impact of each sector on the problem, identifies specific targets for each entity involved and documents the success or failure of each player $(27,28,43)$. Robust monitoring data are needed to support every component. For example, the reduction in dietary salt consumption achieved in the UK (44) required baseline information that enabled targets and action plans to be set for each corporation involved in the production of salty foods as well as regular follow-up data to ensure actions were implemented.

In this vein, the translational objectives of this piece of work will prioritize the collection of those food composition data that will be most readily used to support government policies and industry practices targeting the improvement of the food supply. The provision of outputs from the research that can be used to support grass roots campaigns led by consumer and other advocacy groups will also be a priority, since it is likely that both government and industry can be significantly influenced by community opinion and the media that it generates $(45,46)$.

There are many food composition databases already in existence $(24,47)$, but most provide only summary metrics for groups of similar foods, are substantively incomplete, are out of date, and/or can be accessed only by specific groups or at substantial cost. As such, the capacity of public health researchers and advocates to use food composition data is often significantly constrained. Data describing the composition of individual foods is important because it allows direct comparison of the brands produced by different companies, and can introduce a healthorientated element of competition to the marketplace. Novel mobile technologies have the potential to put this information into the hands of very large numbers of customers, and this may be a further means of pressuring companies into improving the healthiness of their product range. Repeat surveys collecting brand-specific data also enable individual companies to be held to account for how the levels of adverse nutrients in their products are changing over time.

Another important aspect of the approach proposed for the INFORMAS food composition monitoring module is that it will cover as many foods as feasible and not just a sample. The reason for taking a comprehensive approach to data collection, as opposed to sampling, is twofold. First, to bring pressure to bear upon individual corporations requires data on the majority of products marketed by the businesses operating in the field. While data will, of course, never be absolutely complete, experience to date suggests that the approach proposed here can deliver the data required. Second, the considerable public interest in this area, and the demonstrated capacity of crowd-sourcing techniques to collect the data, makes this a situation in 
which complete data collection may be more feasible and cost-effective than sampling, which can add significant additional methodological complexity. Another approach might be to focus on the most widely consumed food products, although the sales volume data required to implement this strategy are rarely available.

The average levels of the measurement indicators, and any difference between them, will mostly be calculated as crude estimates unadjusted for sales volumes because data on sales volumes or other measures of market share are mostly unavailable or expensive to acquire. Prior work where sales volume data have been available has showed that average values based on sales weighted estimates are different to those based on crude unweighted data, although not substantively so (48). Within the proposed programme of work related to this module of INFORMAS, we will seek to identify settings in which sales volume data are available, and better explore the extent to which crude unweighted data are, or are not, a good reflection of population consumption of different nutrients. A broad geographic coverage of countries will be sought with no a priori restriction on participation. The absence of legislation requiring nutrient declarations (and other aspects of food labelling) will initially limit the extent of data collection in some countries, but their participation will be important to achieve these basic regulatory requirements.

There are specific capacity and resource requirements necessary for the conduct of this component of INFORMAS. However, commencement requires rather few resources, and there are many groups around the world able to support countries that wish to embark on this initiative (20). Furthermore, the extent of implementation can be staged with additional elements introduced only when the required resource can be identified. The ready availability of tried and tested tools to support the collection and analysis of data is also a significant advantage, and there is the potential for the component of INFORMAS described here to link to other modules, with sharing of resources likely to generate economies of scale.

\section{Conclusion}

It is long established that the greatly expanded use of processed and restaurant foods has been a key driver in the evolution of the global NCD epidemic (13). Primary vectors for this epidemic are large transnational corporations driven by the shared goal of maximizing shareholder value (10). Unfortunately, the market forces under which these companies operate provide little space for health, and there are powerful commercial factors that drive these companies towards the addition of large quantities of unhealthy nutrients to their products. The purpose of this module, as a part of the broader INFORMAS initiative, is to contribute new data that can help identify solutions and monitor their effectiveness.

The programme of work planned by INFORMAS is unique in that it represents a coordinated global effort to address the upstream determinants of health using objective robust methodologies to track the nature and determinants of the food supply (16). INFORMAS will not deliver instant resolution to the problem, but the data that it provides, and the objective measures it reports, will for the first time hold the food industry directly to account for its actions. It is hoped that this can support the delivery of a more appropriate balance between the legitimate efforts of these corporations to provide and market food products, and the societal imperative that they do it in a way that does not cause massive premature death and disability among the population.

\section{Acknowledgements}

The Rockefeller Foundation kindly supported the work of INFORMAS by hosting the first formal meeting of INFORMAS at the Rockefeller Foundation Bellagio Centre, Italy from 19 to 23 November 2012. The following organizations provided funding support for the travel of participants to Italy for this meeting and the preparation of background research papers: The Rockefeller Foundation, International Obesity Taskforce (IOTF), University of Auckland, Deakin University, The George Institute, University of Sydney, Queensland University of Technology, University of Oxford, University of Pennsylvania Perelman School of Medicine, World Cancer Research Fund International, University of Toronto, The Australian National University. The authors would like to thank Francesco Branca and Godfrey Xuereb from the World Health Organization, and Janice Albert from the Food and Agriculture Organization for their participation in the first formal meeting of INFORMAS, and for their input into this paper. The Faculty of Health at Deakin University kindly supported the costs for open access availability of this paper, and the Australian National Health and Medical Research Council Centre for Research Excellence in Obesity Policy and Food Systems (APP1041020) supported the coordination and finalizing of INFORMAS manuscripts. Bruce Neal is supported by an Australian Research Council Future Fellowship.

\section{Conflicts of interest}

Bruce Neal is the Chair of the Australian Division of World Action on Salt and Health (2007-ongoing), was a Member of the Pepsico Global Scientific Advisory Board (20102012), was the Independent Adjudicator for the Australian Responsible Marketing to Children's Initiative (20092010) and holds funding from the Australian Food and 
Grocery Council as part of a National Health and Medical Research Council of Australia Partnership project (20102014). The other authors declare that they have no competing interests.

\section{References}

1. Popkin BM, Adair LS, Ng SW. Global nutrition transition and the pandemic of obesity in developing countries. Nutr Rev 2012; 70: 3-21.

2. Hawkes C. Uneven dietary development: linking the policies and processes of globalization with the nutrition transition, obesity and diet-related chronic diseases. Global Health 2006; 2: 4 online (18 pp.).

3. Popkin BM, Gordon-Larsen P. The nutrition transition: worldwide obesity dynamics and their determinants. Int J Obes Relat Metab Disord 2004; 28(Suppl. 3): S2-S9.

4. Beaglehole R, Yach D. Globalisation and the prevention and control of non-communicable disease: the neglected chronic diseases of adults. Lancet 2003; 362: 903-908.

5. Ng S, Popkin B. Monitoring foods and nutrients sold and consumed in the United States: dynamics and challenges. J Acad Nutr Diet 2012; 112: 41-45.

6. Hu D, Reardon T, Rozelle S, Timmer CP, Wang H. The emergence of supermarkets with Chinese characteristics: challenges and opportunities for China's agricultural developments. Dev Policy Rev 2004; 22: 557-586.

7. Reardon T, Timmer CP, Minton B. Supermarket revolution in Asia and emerging development strategies to include small farmers. Proc Natl Acad Sci USA 2012; 109: 2332-12337.

8. Slining M, Wen S, Popkin B. Food Companies' CalorieReduction Pledges to Improve U.S. Diet.. Am J Prev Med 2013; 44 (2): 174-184.

9. Lim S, Vos T, Flaxman A et al. The burden of disease and injury attributable to 67 risk factors and risk factor clusters in 21 regions 1990-2010: a systematic analysis for the Global Burden of Disease Study. Lancet 2013; 380: 2224-2226.

10. Moodie R, Stuckler D, Monteiro C et al. Profits and pandemics: preventing the harmful influence of the tobacco, alcohol and ultra processed food and drink industries. Lancet 2013; 381: 670679.

11. Swinburn B, Sacks G, Hall K et al. The global obesity pandemic: shaped by global drivers and local environments. Lancet 2011; 378: 804-814.

12. World Health Organization. Prevention and Control of Noncommunicable Diseases: Implementation of the Global Strategy. Sixty-First World Health Assembly, Resolution WHA61.14. Geneva: World Health Organization, 2007.

13. World Health Organisation. Global Strategy on Diet, Physical Activity and Health. World Health Organization: Geneva, 2004.

14. Lang T, Rayner G, Kaelin E. The Food Industry, Diet, Physical Activity and Health: a review of reported commitments and practice of 25 of the world's largest food companies. Report to World Health Organisation. Centre for Food Policy, City University, London, 2006.

15. Yach D, Lucio A, Barroso C. Can food and beverage companies help improve population health? Some insights from PepsiCo. Med J Aust 2007; 187: 656-657.

16. Swinburn B, Sacks G, Vandevijvere $S$ et al. International Network for Food and Obesity/non-communicable diseases Research, Monitoring and Action Support (INFORMAS): overview and key principles. Obes Rev 2013; 14 (Suppl. 1): 1-12.
17. Greenfield H, Southgate DAT. Food Composition Data Production, Management and Use. Food and Agriculture Organization of the United Nations: Rome, 2003.

18. Elmadfa I, Meyer AL. Importance of food composition data to nutrition and public health. Eur J Clin Nutr 2010; 64(Suppl. 3): S4-S7.

19. Chopra M, Galbraith S, Darnton-Hill I. A global response to a global problem: the epidemic of overnutrition. Bull World Health Organ 2002; 80: 952-958.

20. The Food Monitoring Group. Progress with a global branded food composition database. Food Chem 2013; 140 (3): 451-457. 21. The Food Monitoring Group. International collaborative project to compare and track the nutritional composition of fast foods. BMC Public Health 2012; 12: 559 online (7 pp.).

22. Dunford E, Webster J, Metzler $\mathrm{AB}$ et al. International collaborative project to compare and monitor the nutritional composition of processed foods. Eur J Prev Cardiol 2012; 19: 13261332.

23. Webster JL, Dunford EK, Neal BC. A systematic survey of the sodium contents of processed foods. Am J Clin Nutr 2010; 91: 413-420.

24. Food Standards Agency. Processed Food Databank, 2006. [WWW document]. URL http://www.food.gov.uk/science/ surveillance/fsisbranch2006/fsis1306 (accessed 12 November 2009).

25. Grimes GA, Nowson CA, Lawrence M. An evaluation of the reported sodium content of Australian food products. Int J Food Sci Technol 2008; 43: 2219-2229.

26. Guest G, MacQueen K, Namey E. Applied Thematic Analysis. Sage publications: Thousand Oaks, CA, 2012.

27. Webster JL, Dunford EK, Hawkes C, Neal BC. Salt reduction initiatives around the world. J Hypertens 2011; 29: 10431050 .

28. Webster J, Dunford E, Kennington S, Neal B, Chapman S. Drop the Salt! Assessing the impact of a public health advocacy strategy on government policy in Australia. Publ Health Nutr 2012; 22: 1-7.

29. Rayner M, Wood AT, Lawrence M et al. Monitoring the health-related labelling of foods and non-alcoholic beverages in retail settings. Obes Rev 2013; 14 (Suppl. 1): 70-81.

30. Lee A, Ni Mhurchu C, Sacks G et al. Monitoring the price and affordability of foods and diets globally. Obes Rev 2013; 14 (Suppl. 1): 82-95.

31. Food Standards Australia New Zealand. P230: Consideration of Mandatory Fortification with Iodine, 2007. [WWW document]. URL http://www.foodstandards.gov.au/code/proposals/Pages/ proposalp230iodinefo2802.aspx (accessed 13 February 2013).

32. The European Food Information Resource E. The EuroFIR Project - A story of success, 2010. [WWW document]. URL http:// www.eurofir.net/ (accessed 20 January 2013).

33. Food and Agriculture Organization of the United Nations. Background to INFOODS, 2010. [WWW document]. URL http:// www.fao.org/infoods/index_en.stm (accessed 20 January 2013). 34. Food Standards Agency. Processed food databank: Sampling round two: February 2007-September 2007, 2008. [WWW document]. URL http://www.food.gov.uk/multimedia/pdfs/fsis0108.pdf (accessed 20 January 2013).

35. New York City Department of Health and Mental Hygiene. National Salt Reduction Initiative, 2010. [WWW document]. URL http://www.nyc.gov/html/doh/html/cardio/cardio-saltinitiative.shtml (accessed 12 Jan 2011).

36. Codex Alimentarius. Guidelines on Nutrition Labelling, 2011. [WWW document]. URL http://www.codexalimentarius.org/input/ download/standards/34/CXG_002e.pdf (accessed 12 July 2012). 
37. Webster J, Dunford E, Neal B. A systematic survey of the sodium contents of processed foods. Am J Clin Nutr 2010; 91 (2): 413-420. doi: 10.3945/ajen.2009.28688.

38. Dunford E, Webster J, Metzler AB et al. International collaborative project to compare and monitor the nutritional composition of processed foods. Eur J Cardiovasc Prev Rehabil 2012; 19: $1326-1332$.

39. Dunford EK, Eyles H, Mhurchu CN, Webster JL, Neal BC. Changes in the sodium content of bread in Australia and New Zealand between 2007 and 2010: implications for policy. Med J Aust 2011; 195: 346-349.

40. Dunford E, Webster J, Woodward M et al. The variability of reported salt levels in fast foods across six countries: opportunities for salt reduction. Can Med Assoc J 2012; 184: 1023-1028.

41. Regional Expert Group for Cardiovascular Disease Prevention through Population-wide Dietary Salt Reduction - Sub-group on industry liaison. A Guide for Setting Targets and Timelines to Reduce the Salt Content of Food. Pan American Health Organisation: Washington DC, 2013.

42. Cobiac LJ, Vos T, Veerman JL. Cost-effectiveness of interventions to reduce dietary salt intake. Heart 2010; 96: 1920-1925. 43. Webster J, Dunford E, Huxley R, Li N, Nowson C, Neal B. The development of a national salt reduction strategy for Australia. Asia Pac J Clin Nutr 2009; 18: 303-309.
44. Wyness L, Butriss L, Stanner S. Reducing the population's sodium intake: the UK Food Standards Agency's salt reduction programme. Publ Health Nutr 2012; 15: 254-261.

45. Sandman PM. Hazard versus outrage in the public perception of risk. In: Covello VT, McCallum DB, Pavlova MT (eds). Effective Risk Communication: The Role and Responsibility of Government and Nongovernment Organizations. Plenum Press: New York, 1989, pp. 45-49.

46. Chapman S, Freeman B. Markers of the denormalisation of smoking and the tobacco industry. Tob Control 2008; 17: $25-31$.

47. Center for Science in the Public Interest. Transatlantic Cooperation on Food Marketing, Labeling, \& Nutrition Urged to Help Curb Obesity Epidemic, 2005. [WWW document]. URI http://www.cspinet.org/new/200511281.html (accessed 1 September 2012).

48. Institute of Medicine. Strategies to reduce sodium intake in the United States. Washington, United States, 2010. [WWW document]. URL http://www.iom.edu/Reports/2010/Strategies -to-Reduce-Sodium-Intake-in-the-United-states.aspx (accessed 12 January 2013). 